\title{
Die Flughäfen der Schweiz
}

\author{
Ulrich Haller
}

\section{Einleitung}

Die Geographie hat bisher das Studium der Auswirkungen der Luftfahrt auf die Landschaft weitgehend vernachlässigt. Dabei sind doch beispielsweise die direkten und indirekten Einflüsse der Luftfracht auf die Landschaft des mittleren und unteren Glattales schlechthin dominierend. Die Bedeutung des Luftverkehrs scheint während langer Zeit auch von den Regionalplanern unterschätzt worden zu sein. Die ungeregelte Überbauung im Nahbereich der Flughäfen, ja teilweise direkt unter dem Anflugsektor der Hauptpisten, nach der Erstellung der Flughäfen, hat in zahlreichen Fällen (z. B. Kloten, London-Heathrow) negative Folgen gezeitigt.

Welche Auswirkungen der Bau eines neuen Großflughafens haben kann, zeigen neue Untersuchungen, die in England im Zusammenhang mit der Standortsplanung für einen dritten Londoner Flughafen - neben Heathrow und Gatwick - angestellt wurden (6). So wird damit gerechnet, daß etwa 70000 Personen auf diesem Flughafen Beschäftigung finden werden. Mit ihren Familien werden es also rund 200000 Personen sein, zu deren Versorgung nochmals 100000 Personen kommen. Wenn man also von einem dritten Londoner Flughafen spricht, sollte gleichzeitig eine neue Stadt in der Größenordnung von Genf in die Planung einbezogen werden.

Die Bedeutung des Luftverkehrs geht aus der Tatsache hervor, daß die Luftfahrt auf vielen Strecken seit Jahren ein höheres Passagieraufkommen verzeichnet als die übrigen Verkehrsträger (Beispiel: Nordatlantik). Auch im Luftfrachtverkehr über Langstrecken dürften die Zahlen der bodengebundenen Verkehrsmittel eines Tages übertroffen werden, wenn von der Beförderung von Massengütern abgesehen wird. Für das Binnenland Schweiz wird der Luftverkehr in noch verstärktem Maße als bisher an Bedeutung für die gesamte Wirtschaft gewinnen.

\section{Technische Voraussetzungen}

Der Bau der Flughäfen wird von der Internationalen Zivilluftfahrtsorganisation ICAO (International Civil Aviation Organization) reglementiert. Die fol- genden Faktoren müssen unter anderem beim Bau eines Flughafens grundsätzlich berücksichtigt werden (3):

Das Pistensystem soll in den vorherrschenden Windrichtungen angelegt werden, da die Flugzeuge wohl starke Winde von vorn, jedoch nur Seitenwinde von geringerer Stärke ertragen. Die zulässigen Windgeschwindigkeiten dürfen beispielsweise beim Start eines Langstreckenflugzeuges des Typs DC-8 maximal $110 \mathrm{~km} / \mathrm{h}$ von vorne, jedoch nur $63 \mathrm{~km} / \mathrm{h}$ aus einem Winkel von $90^{\circ}$ zur Startrichtung betragen.

Die Pistenlänge richtet sich nach den zur Verwendung gelangenden Flugzeugtypen, der Höhe über Meer (geringere Luftdichte hat längere Startstrekken zur Folge) und den auftretenden Lufttemperaturen (höhere Lufttemperaturen bewirken ein Absinken der Schubkraft der Strahltriebwerke).

Die Umgebung des Flughafens muß frei von Hindernissen sein. Besonders strenge Bedingungen in bezug auf Hindernisfreiheit werden bei den Anflugsektoren und den Startsegmenten in der Verlängerung der Pistenachse gestellt, da Start und Landung heute auch bei klarem Wetter weitgehend nach Instrumenten erfolgen. Bei einer Hauptstartpiste besteht das Startsegment beispielsweise aus einer Fläche, die vom Pistenende bis in eine Horizontaldistanz von $15 \mathrm{~km}$ mit einer Neigung von $2 \mathrm{Grad}$ ansteigt. Die Breite dieses Segmentes, in das keine Hindernisse hineinragen dürfen, beträgt am Pistenrand $180 \mathrm{~m}$. Mit zunehmender Entfernung vom Pistenrand verbreitert sich das Segment, bis schließlich eine Breite von $1200 \mathrm{~m}$ erreicht ist. Diese strengen Vorschriften haben in vielen Fällen zur Folge, daß Wald gerodet und Hügel abgetragen werden müssen. Es sind also nicht nur die Pistensysteme, welche eine Veränderung des Landschaftsbildes bewirken, auch die umliegenden Landschaften werden umgestaltet.

Bedeutend weniger streng sind die Vorschriften bei den kleinen Flugplätzen und Flugfeldern, bei denen Anflug und Abflug im Sichtflug erfolgen.

\section{Die Schweizer Flughäfen und Flugplätze}

Mit Zürich-Kloten und Genf-Cointrin besitzt die Schweiz zwei Interkontinentalflughäfen der höchsten Klasse, die von allen gegenwärtig verwendeten 


$\begin{array}{llll}\text { Flugplatz } & \text { Höhe ü. M. } & \begin{array}{l}\text { Abmessungen } \\ \text { der Pisten } \\ \text { in } \mathrm{m}\end{array} & \begin{array}{l}\text { Oberfläche } \\ \text { B Beton } \\ \text { As Asphalt }\end{array} \\ & \text { G Gras }\end{array}$

\begin{tabular}{|c|c|c|c|c|}
\hline Altenrhein & 398 & $\begin{array}{r}1200 \times 30 \\
600 \times 30\end{array}$ & As & $\begin{array}{l}\text { Werkflugplatz der } \\
\text { Flugzeugwerke }\end{array}$ \\
\hline Amlikon & 420 & $800 \times 30$ & G & Nur Segelflug \\
\hline Ascona & 200 & $625 \times 20$ & As & \\
\hline Bad Ragaz & 492 & $495 \times 12$ & As & \\
\hline \multirow[t]{3}{*}{ Basel-Mülhausen } & 269 & $2370 \times 60$ & B & Flughafen des \\
\hline & & $1600 \times 60$ & As & Linienverkehrs \\
\hline & & $650 \times 40$ & G & \\
\hline Bellechasse & 432 & $530 \times 50$ & G & \\
\hline \multirow[t]{2}{*}{ Bern-Belp } & 510 & $1310 \times 30$ & As & Flughafen mit \\
\hline & & $650 \times 30$ & G & beschränktem Linienverkehr \\
\hline Bex & 410 & $700 \times 30$ & G & \\
\hline Biel-Kappelen & 438 & $630 \times 30$ & G & \\
\hline Birrfeld & 400 & $600 \times 40$ & G & \\
\hline La Côte & 412 & $630 \times 40$ & G & \\
\hline Courtelary & 690 & $580 \times 30$ & G & \\
\hline Dittingen & 555 & $600 \times 30$ & G & \\
\hline Ecuvillens & 700 & $637 \times 30$ & $\mathrm{G}$ & \\
\hline Les Eplatures & 1020 & $740 \times 30$ & As & \\
\hline Fricktal-Schupfart & 545 & $600 \times 30$ & $\mathrm{G}$ & \\
\hline \multirow[t]{2}{*}{ Genève-Cointrin } & 430 & $3900 \times 50$ & $\mathrm{~B}$ & Flughafen des \\
\hline & & $615 \times 30$ & G & Linienverkehrs \\
\hline \multirow[t]{2}{*}{ Grenchen } & 430 & $580 \times 30$ & G & \\
\hline & & $380 \times 30$ & G & \\
\hline Gruyères & 688 & $710 \times 30$ & G & \\
\hline Hasenstrick & 760 & $400 \times 30$ & G & Nur beschränkt benützbar \\
\hline Hausen am Albis & 590 & $700 \times 30$ & G & \\
\hline Langenthal & 480 & $550 \times 30$ & G & \\
\hline Lausanne-Blécherette & 623 & $845 \times 100$ & G & \\
\hline \multirow[t]{4}{*}{ Locarno } & 200 & $700 \times 60$ & G & Gemeinsam mit Flugwaffe \\
\hline & & $700 \times 80$ & G & \\
\hline & & $700 \times 60$ & G & \\
\hline & & $600 \times 30$ & G & \\
\hline Lommis & 469 & $650 \times 30$ & G & \\
\hline \multirow[t]{2}{*}{ Lugano } & 278 & $1200 \times 30$ & As & \\
\hline & & $600 \times 30$ & G & \\
\hline Luzern-Beromünster & 650 & $540 \times 30$ & G & \\
\hline Mex & 480 & $500 \times 30$ & G & \\
\hline Môtiers & 732 & $550 \times 30$ & $\mathrm{G}$ & \\
\hline \multirow[t]{2}{*}{ Neuchâtel } & 435 & $700 \times 20$ & As & \\
\hline & & $550 \times 30$ & G & \\
\hline Olten & 415 & $560 \times 30$ & G & Gesperrt für auswärtige Piloten \\
\hline Porrentruy & 440 & $550 \times 30$ & G & \\
\hline Samedan & 1710 & $1800 \times 40$ & As & Gemeinsam mit Flugwaffe \\
\hline Schaffhausen & 465 & $400 \times 30$ & $\mathrm{G}$ & nur Segelflug \\
\hline Schänis & 416 & $600 \times 30$ & G & nur Segelflug \\
\hline Schmitten & 600 & $450 \times 30$ & G & Für auswärtige Piloten gesperrt \\
\hline \multirow[t]{2}{*}{ Sion } & 482 & $2000 \times 40$ & As & Gemeinsam mit Flugwaffe \\
\hline & & $950 \times 30$ & G & \\
\hline Sitterdorf & 507 & $600 \times 30$ & G & \\
\hline Speck & 535 & $700 \times 30$ & G & \\
\hline
\end{tabular}




$\begin{array}{lllll}\text { Thun } & 560 & 700 \times 150 & \text { G } & \text { Während Wochentagen } \\ & & 600 \times 160 & \text { G } & \text { gesperrt } \\ \text { Triengen } & 490 & 419 \times 15 & \text { As } & \\ \text { Wangen-Lachen } & 407 & 500 \times 18 & \text { As } & \\ \text { Winterthur } & 457 & 600 \times 30 & \text { G } & \text { Nur Segelflug } \\ \text { Yverdon } & 440 & 850 \times 30 & \text { G } & \\ \text { Zürich-Kloten } & 432 & 3700 \times 60 & \text { B } & \text { Flughafen des } \\ & & 2500 \times 60 & \text { B } & \text { Linienverkehrs } \\ & 1535 \times 45 & \text { B } & \\ & 500 \times 50 & \text { G } & \end{array}$

zivilen Flugzeugtypen benützt werden können. Als Folge der wechselnden Windrichtungen mußten in Zürich-Kloten zwei Hauptpisten gebaut werden. Eine weitere Landepiste mit einer Länge von $3300 \mathrm{~m}$ ist projektiert. Die aus der ersten Bauperiode stammende dritte Piste, die sogenannte Bisenpiste, wird heute nur noch wenig benützt. Viel günstiger sind die Verhältnisse in Genf-Cointrin. Da die Winde durch die umliegenden Juraketten kanalisiert werden, genügt vorläufig noch eine einzige Piste. Um sie auf die notwendige Länge ausbauen zu können, mußte ein Gebietsabtausch zwischen Frankreich und der Schweiz vorgenommen werden.

Auch der Bau des Flughafens Basel-Miilhausen bedingte den Abschluß von verschiedenen Staatsverträgen, liegt doch das gesamte Flughafenareal auf französischem Gebiet in der oberrheinischen Tiefebene. Die vorhandenen Pisten lassen die Verwendung von modernen Langstreckenflugzeugen nur unter gewissen Einschränkungen zu. Da der Flughafen Basel relativ spät in Betrieb genommen werden konnte und das Verkehrsaufkommen der Region Basel kleiner ist als jenes von Zürich oder Genf, blieb der Linienverkehr in Basel wesentlich geringer. 1967 verteilte sich der gesamte Personenverkehr der drei großen Flughäfen folgendermaßen (5): Zürich: 56,2\%, Genf: 33,2\%, Basel: $10,6 \%$. Zürich lag dabei in Europa hinter London, Paris, Frankfurt, Rom, Kopenhagen, Berlin und Amsterdam an achter, Genf an zwanzigster Stelle. Der Flughafen Bern-Belp wird nur von relativ kleinen Verkehrsflugzeugen der Balair/Swissair und von verschiedenen Chartergesellschaften angeflogen, da die Pistenlänge und vor allem die Hindernisfreiheit nicht dem internationalen Standard entsprechen. Eine Verlängerung der Piste in Belp dürfte nur zu einer bescheidenen Verbesserung der Verhältnisse führen, da die Topographie ungünstig ist. Obwohl verschiedene Studien über andere Flughafenstandorte nicht ungünstige Resultate ergeben haben, ist die Bedürfnisfrage für einen Kontinentalflughafen Bern nicht abgeklärt (4).
Unter den übrigen Flugplätzen der Schweiz sind gegenwärtig nur noch jener von Sion und in beschränktem Maße auch Altenrhein in der Lage, kleine Verkehrsflugzeuge aufzunehmen. Außerdem bestehen noch zahlreiche Militärflugplätze, die teilweise über kilometerlange Hartbelagpisten verfügen, jedoch nur in Ausnahmefällen von zivilen Flugzeugen angeflogen werden dürfen. Bei den restlichen Flugplätzen und Flugfeldern handelt es sich meistens um einfache Anlagen mit kurzen Pisten, die nur tagsüber und bei gutem Flugwetter von Leichtflugzeugen benützt werden können. Sie dienen vorwiegend der Ausbildung von Zivil- und Militärpiloten. Außerdem sind auf diesen Plätzen die zahlreichen privaten Sport-, Reise- und Segelflugzeuge stationiert (Bestand am 1. Januar 1969 (7): 870 Leichtflugzeuge und 370 Segelflugzeuge).

\section{Ausblick}

Mit zwei Interkontinentalflughäfen und einem Kontinentalflughafen besitzt die Schweiz eine überdurchschnittlich hohe Flughafendichte, verfügen doch beispielsweise Belgien, die Niederlande, Dänemark und Schweden nur über je einen einzigen Interkontinentalflughafen. Durch den fortschreitenden Ausbau der Nationalstraßen schrumpfen die Zubringerzeiten aus den meisten Regionen der Schweiz auf Werte zusammen, die denjenigen entsprechen, die heute in ausländischen Großstädten für die Fahrt vom Stadtzentrum zum Flughafen benötigt werden. Der Bau von weiteren Großflughäfen dürfte sich daher erübrigen.

Verschiedene Projekte sehen den Bau von mittelgroßen Kontinentalflughäfen vor, die auch von Flugzeugen der Charterflugggesellschaften, dem wichtigsten Transportmittel des modernen Massentourismus, angeflogen werden können. In erster Linie ist in diesem Zusammenhang der Bau eines neuen Flughafens Lausanne in Etagnières zu nennen. 


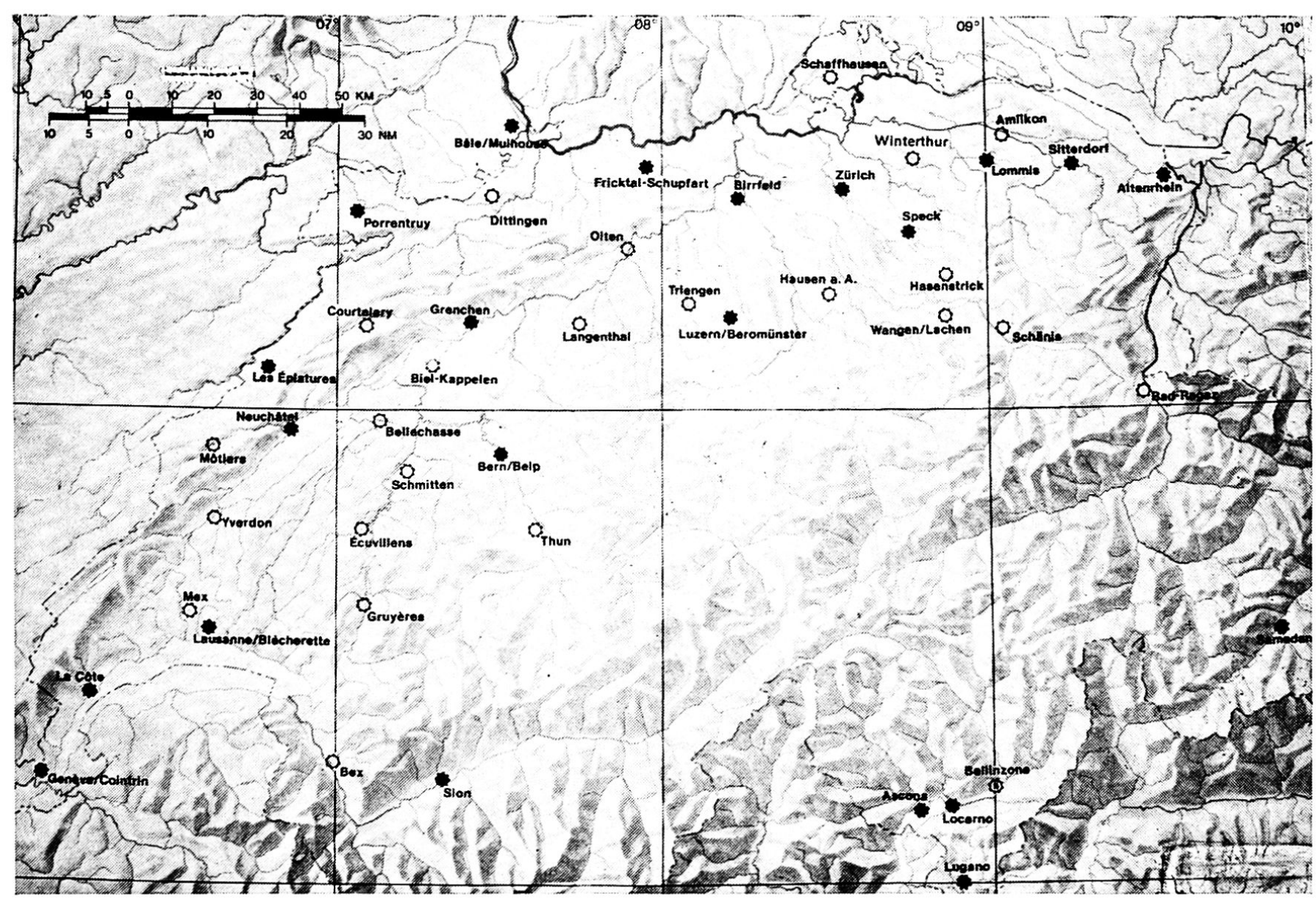

Die Flugplätze der Schweiz. Ausgefüllte Ringe: Zollflugplätze; Ring mit H: Helikopterflugplatz

Flugplätze werden sicher auf Jahrzehnte hinaus weiterhin nötig sein. Ausgedehnte kommerzielle Versuche mit Senkrechtstartern (Helikoptern) in New York, Los Angeles und den Beneluxstaaten sind aus wirtschaftlichen und technischen Gründen weitgehend gescheitert. Die Entwicklung scheint vor allem im Zubringerverkehr eher in Richtung auf betriebssichere Kurzstartflugzeuge zu gehen, die jedoch immer noch gut ausgebaute Flugplätze mit Pistenlängen von 500-1000 m erfordern.

Die Schaffung neuer Flugplätze oder der Ausbau bestehender Anlagen stößt jedoch in unserem dicht besiedelten Land auf größte Schwierigkeiten. Verschiedene günstig gelegene Sportflugplätze sind im Gegenteil von den wachsenden Siedlungen verschluckt worden (Sisseln, Spreitenbach, Biel-Bözingen). Vor allem in der Region Zürich fehlt es heute an einem geeigneten Flugplatz für Leichtflugzeuge, da Kloten für diese Flugzeugkategorie nur beschränkt benützbar ist. Es zeigt sich hier das große Dilemma der modernen Luftfahrt: Die Flugplätze sollten möglichst in der Nähe jener Siedlungen liegen, denen sie dienen. Anderseits ist gerade dort die Anlage von Flugplätzen aus bekannten Gründen
(Lärm, Bedarf an großen, meist günstig gelegenen Arealen) nicht erwünscht. Es wäre eine interessante Aufgabe der Regionalplanung, hier optimale Lösungen zu finden, die den Interessen aller Beteiligten dienen.

\section{Verwendete Literatur}

1 AIP Switzerland, herausgegeben vom Eidgenössischen Luftamt, Bern.

2 Allen, Roy: Große Flughäfen der Welt. Zürich 1968.

3 Fäßler, E.: Vorschriften für die Planung eines Flughafens. NZZ Nr. 529, 1968.

4 Meyer, Arnold: Städteflughafen Bern. Berner Beiträge zur Nationalökonomie. Bern 1966.

5 Jahresberichte der Flughäfen Basel, Genf und Zürich.

6 Mitteilungen in Flight International und Financial Times.

7 Schweizerisches Luftfahrzeugregister, Eidg. Luftamt, Bern. 